\title{
Inteligencia artificial en educación: una revisión de la literatura en revistas científicas internacionales
}

\author{
Artificial intelligence in education: a review of the literature in \\ international scientific journals
}

\author{
Fernando Alain Incio Flores, ${ }^{\text {1a }}$ Dulce Lucero Capuñay Sanchez, ${ }^{2}$ Ronald Omar Estela Urbina, ${ }^{3}$ \\ Miguel Ángel Valles Coral, ${ }^{4}$ Segundo Edilberto Vergara Medrano, ${ }^{5}$ \\ Duberli Geomar Elera Gonzales ${ }^{6}$ \\ Universidad Nacional Intercultural Fabiola Salazar Leguía de Bagua, Perú ${ }^{13}$ \\ Universidad Cesar Vallejo, Lima, Perú ${ }^{2}$ \\ Universidad Nacional de San Martín, San Martín, Perú ${ }^{4}$ \\ Universidad nacional Intercultural de la Amazonía, Perú ${ }^{5}$ \\ Universidad Nacional Autónoma de Chota, Cajamarca, Perú ${ }^{6}$ \\ (iD) ORCID ID: https://orcid.org/0000-0003-3286-7787 ${ }^{1}$ \\ (iD) ORCID ID: https://orcid.org/0000-0001-8678-5766 $^{2}$ \\ iD ORCID ID: https://orcid.org/0000-0001-5240-1242 3 \\ iD ORCID ID: https://orcid.org/0000-0002-8806-2892 ${ }^{4}$ \\ iD ORCID ID: https://orcid.org/0000-0001-7611-3827 \\ (iD) ORCID ID: https://orcid.org/0000-0001-9854-840X
}

Recibido: 27 de junio de 2021

Aceptado: 07 de octubre de 2021

\section{Resumen}

Esta investigación consiste en una revisión literaria de publicaciones científicas en el área de la inteligencia artificial (IA), pertenecientes a revistas científicas encontradas en el portal SCImago Journal \& Country Rank. La búsqueda de información se realizó utilizando palabras clave y títulos de investigaciones publicadas entre los años 1970 y 2020 en la base de datos de Scopus. El objetivo de este artículo es identificar los aportes de la IA en la educación en las últimas cinco décadas, dara conocer las revistas científicas con los índices de impacto más altos en el área de la IA en los últimos 10 años, y analizar el papel que desempeñará la IA en la educación post Covid-19. Los resultados evidencian aportes significativos de la IA en la educación, empleando técnicas de redes neuronales, big data, visión por computador, asistentes digitales virtuales, aprendizaje automático y análisis predictivo, siendo Estados Unidos el país que posee el mayor número de revistas científicas (siete) dedicadas al área de la IA. Finalmente, destacamos la necesidad de involucrar la IA en el proceso de enseñanza y aprendizaje en una educación post Covid-19.

Palabras clave: inteligencia artificial, educación, enseñanza, aprendizaje, Covid-19, tecnología

${ }^{\mathrm{a} C}$ Correspondencia al autor:

E-mail: fincio@unibagua.edu.pe 


\begin{abstract}
This research consists of a literary review of scientific publications in the area of Artificial Intelligence, belonging to scientific journals found in the SCImago Journal \& Country Rank portal. The information search was carried out using keywords and titles of research published from 1970 to 2020 in the Scopus database. The objective of this article is to identify the contributions of AI to education over the last five decades, to present the scientific journals with the highest impact rates in the area of AI over the last 10 years and to analyse the role that AI will play in post-Covid19 education. The results show significant contributions of the Artificial Intelligence in education using neural network techniques, big data, computer vision, virtual digital assistants, automatic learning, language processing and predictive analysis. The United States has the largest number of scientific journals (seven) dedicated to the area of AI. Finally, we highlight the need to involve AI in the teaching and learning process in a post Covid-19 education.
\end{abstract}

Keywords: artificial intelligence, education, teaching, learning, Covid-19, technology.

\title{
Introducción
}

Esta investigación consiste en una revisión bibliográfica enmarcada en el campo de la Inteligencia Artificial (IA) y la educación, basada en el análisis documental de artículos científicos comprendidos entre los años 1970 al 2020 y en la búsqueda de revistas científicas en el portal SCImago Journal \& Country Rank a partir del año 2010. En definición, la IA es la capacidad que tiene un sistema computacional para simular el comportamiento del cerebro humano, capaz de recibir datos externos en calidad de información, aprender mediante el entrenamiento y, en base a este aprendizaje, lograr los objetivos para el que fue entrenado (Brazdil \& Jorge, 2001). Desde que el término "inteligencia artificial" fue acuñado en la década de los 50 (Aranda-Corral, Calmet \& Martín-Mateos, 2014), se han esperado importantes aportes en diversas áreas: “la robótica, la visión de bajo nivel, el procesamiento del lenguaje natural, y los sistemas expertos basados en el conocimiento y sus usos para la educación, la ciencia y la tecnología" (Boden, 1984, p. 60).

Por otro lado, los aportes de la IA a la educación son numerosos y aumentaron progresivamente desde su aparición. En distintas ocasiones fue blanco de críticas al ser abordada por la neurociencia o la psicología educativa, despertando estas inquietudes en algunos críticos: ¿cuál es el papel apropiado de la IA en la educación? (Cukurova, Kent \& Luckin, 2019), ¿debería ser una tecnología de reemplazo humano o una tecnología de asistencia humana? (Cukurova, Luckin \& Clark-Wilson, 2019); sin embargo, otros investigadores destacan lo beneficioso que resulta la IA en el sector educativo, ya sea en el proceso de enseñanza y aprendizaje o en la 
investigación (Cukurova, Luckin, \& Kent, 2020). No solo nos ha brindado herramientas tecnológicas en estos tiempos de pandemia para llevar a cabo las actividades educativas, también nos permite incursionar en otras ciencias como la medicina, ingeniería, biología, economía, entre otras disciplinas (Luckin \& Cukurova, 2019).

En este contexto, ciertas investigaciones realizadas por Boden (1984), Brown y Burton (1978) y Brusilovsky y Peylo (2003) señalan a la IA como un aliado clave en el proceso de enseñanza y aprendizaje en la educación; además, la consideran como una herramienta para garantizar el logro de competencias en los estudiantes (Castrillón, Sarache \& Ruiz-Herrera, 2020; Goldstein \& Papert, 1977). En tal sentido, debería ser considerada como una línea de investigación en estudios de pregrado y postgrado en las instituciones educativas.

Cabe resaltar que, sin la IA, hubiera sido imposible poner en marcha la enseñanza virtual o la enseñanza no presencial que los gobiernos han puesto en práctica. "Así como la enseñanza se materializa en la docencia, se refleja en el aprendizaje y hace parte de la génesis de las universidades" (Benítez-Restrepo, 2020, p. 4), esta se ve influenciada por una serie de factores de orden sociocultural (Cerda et al., 2019), económico (Incio et al., 2021), político (González-García et al., 2019), cognitivo (Guzmán-Zamora \& Gutiérrez-García, 2020) y emocional (Chacón-Vargas \& Roldán-Villalobos, 2021; Pinto, Fernández, \& Cabezas, 2020).

A la luz de estos antecedentes, se considera la importancia y la necesidad de implementar un sistema educativo con soporte tecnológico, el cual incluya a la IA como herramienta de gestión administrativa, académica y de investigación, que permita afrontar temas relacionados a deserción estudiantil, rendimiento académico, proceso de enseñanza y aprendizaje, tutorías inteligentes, inclusión social, educación intercultural, entre otros. Se evidencia así la necesidad de implementar en todos los países un ministerio de ciencia, tecnología e innovación tecnológica como aliado estratégico de la educación.

Por tanto, las preguntas que conducen está investigación son: ¿qué países en el mundo cuentan con revistas científicas en el área de la IA que aporten conocimientos científicos y tecnológicos a la educación?, ¿cuál es el índice de impacto en la comunidad científica que tienen estás revistas desde su creación?, ¿puede la IA ayudar a implementar una educación virtual de calidad?, ¿cuál es el papel que desempeñará la IA en la educación post Covid-19?, ¿cómo el uso y la apropiación de la IA en la educación permite repensar escenarios educativos en tiempos de emergencia? De esta manera, el presente artículo se pretende dar a conocer las respuestas a estas 
preguntas en cuatro secciones: metodología, resultados, discusión y, finalmente, las conclusiones de la investigación.

\section{Metodología}

Para el desarrollo de esta investigación, se utilizó la revisión de literatura como método para identificar, evaluar y sintetizar el conjunto de conocimientos generados por investigadores, académicos o prácticos (Booth, Sutton \& Papaioannou, 2021; Randolph, 2019). Como primera actividad se realizó una revisión de literatura de revistas científicas encontradas en el portal SCImago Journal \& Country Rank, considerando dos aspectos principales: 1) revistas científicas que aborden temas de IA relacionados a educación, y 2) publicaciones accesibles para la investigación. Como segunda actividad se realizó la selección de la literatura a utilizar, a fin de analizar los aportes de la IA en la educación en las últimas cinco décadas. La búsqueda se realizó con los siguientes términos: "Artificial Intelligence”, “Artificial Intelligence AND Education”, "Artificial Intelligence AND Education AND Pandemic", "Neural Networks AND Education", "Expert Systems AND Education", "Diffuse Logic AND Education” y "Artificial Intelligence AND Education AND Expert Systems AND Neural Networks". Cada uno de estos se escribió de manera reiterativa, buscando información durante las últimas cinco décadas. Se eligió este periodo debido a que es un tiempo de considerable aumento en la producción de investigaciones relacionadas a IA y educación. Como tercera actividad se procedió a seleccionar la información comseguida: en una primera selección se revisó el título y resumen de cada artículo científico, teniendo en cuenta el propósito de esta investigación, obteniendo de esta manera 70 trabajos disponibles; en una segunda y definitiva selección, se identificaron y sistematizaron resúmenes, resultados, discusiones y conclusiones de cada uno de los artículos obtenidos en la primera selección, de los cuales finalmente se eligieron 40 de ellos. Para conocer los índices de impacto de las revistas científicas en los últimos 10 años, se acudió a la base de datos de SCImago Journal \& Country Rank. Finalmente, para lograr los objetivos trazados en la investigación, se organizó la información en categorías y subcategorías. 


\section{Tabla 1}

Categorías de estudio

\begin{tabular}{|c|c|}
\hline Categoría & Subcategoría \\
\hline $\begin{array}{lll}\begin{array}{l}\text { Inteligencia } \\
\text { educación }\end{array} & \text { artificial } & \text { y } \\
& & \end{array}$ & $\begin{array}{l}\text { - Investigaciones agrupadas en décadas. } \\
\text { - Investigaciones que involucren redes neuronales, } \\
\text { big data, asistentes digitales virtuales, visión por } \\
\text { computador, aprendizaje profundo, aprendizaje } \\
\text { automático. }\end{array}$ \\
\hline $\begin{array}{l}\text { Revistas científicas en el área } \\
\text { de la IA encontradas en el } \\
\text { portal SCImago Journal \& } \\
\text { Country Rank }\end{array}$ & $\begin{array}{l}\text { - Número de revistas científicas por año. } \\
\text { - Países con revistas científicas en el área de la IA. } \\
\text { - Revistas científicas en el área de la IA que no } \\
\text { están en el ranking por cuartiles. } \\
\text { - Número de revistas por cuartiles. } \\
\text { - Número de documentos por año. }\end{array}$ \\
\hline
\end{tabular}

Fuente: elaboración propia

\section{Resultados}

\section{Inteligencia artificial y educación}

En la década de los 70 e inicio de los 80 , se evidenciaron modelos estudiantiles para el aprendizaje asistido por computador que, a pesar de los resultados decepcionantes por las dificultades y deficiencias, se evidenciaba signos de un futuro dependiente de la IA. En matemáticas, la IA permitió la demostración de teoremas mediante la creación de software matemáticos (Brown \& Burton, 1978), facilitó la creación de entornos educativos en teoría informática (Chandrasekaran, 1975), implementó sistemas de tutoría inteligente (Gable y Page, 1980), e incluso Goldstein y Papert (1977), Rowe (1980) y Self (1979) la consideraron como parte del campo de estudio de la psicología. Durante la década de los 80 e inicio de los 90, se implementó el uso de sistemas expertos para el diagnóstico y evaluación educativa y se propuso integrar aplicaciones y metodologías de la IA en el plan de estudio de las universidades (Boden, 1984). Este periodo también se caracterizó por la creación de entornos de aprendizajes (Jones, 1985), la utilización de sistemas expertos y redes neuronales artificiales (Naughton, 1987), el empleo de 
algoritmos genéticos y lógica difusa en investigaciones de educación, ciencia y tecnología (Salchenberger, 1989), todos ellos considerados técnicas de la IA.

Durante los 90, investigadores en el campo de la IA extendieron sus estudios a dominios complejos, como lenguajes de programación, matemáticas, medicina, física, solución de problemas de aviónica, fábricas de pulpa, fábricas de papel y electrónica (Wasson, 1997). Paralelamente, las facultades de medicina abren discusiones sobre la utilización de la inteligencia artificial en el diagnóstico y tratamiento de enfermedades, argumentando que ella destaca la capacidad de mejorar el aprendizaje de los estudiantes de medicina en numerosos dominios, por lo que debería emplearse para fortalecer las prácticas educativas (Lillehaug \& Lajoie, 1998). Cabe mencionar la aparición de software artificialmente inteligente para ayudar en el aprendizaje a niños autistas mudos (Coldwell, 1995), así como experimentos relacionados al rendimiento académico y el logro de aprendizajes (Benkovič et al., 1997), agrupando estudiantes cuya metodología de enseñanza involucraba o era ajena a la IA (Pham \& Pham, 1999).

A partir del año 2000 hasta la actualidad, se puede ver con más frecuencia el aporte que realiza la IA en la educación, con la implementación de sofisticados sistemas de tutoría inteligente (Amershi et al., 2005; Arevalillo-Herráez, Moreno-Picot, \& Cavero-Millán, 2011), el diseño de video juegos educativos (Brusilovsky \& Peylo, 2003; Cukurova et al., 2020) y la incursión de sistemas de gestión de aprendizaje (Garrido, 2010). Desafortunadamente, aún existen instituciones educativas que mantienen desconectada la educación con la tecnología (Brazdil y Jorge, 2001).

Por otro lado, es evidente la producción de investigaciones realizadas en universidades en las que se proponen mecanismos para la formación docente de modo contínuo e intensivo haciendo uso de la IA. Términos como big data empiezan a ser considerados útiles para lograr el desarrollo sostenible e inclusivo en la educación. Investigadores como Matas-Terrón et al. (2020) exigen que los educadores en proceso de formación se familiaricen en el manejo de estas herramientas, puesto que el educador del futuro tendrá que manejar e integrar la IA en una educación post Covid-19. En esta última década, se ha obtenido resultados satisfactorios en investigaciones que involucran procesos educativos que utilizaron tecnologías basadas en la IA, entre ellas destacan la motivación del aprendizaje (Moreira et al., 2019), mejora del rendimiento académico (Castrillón et al., 2020), prevención de la deserción escolar (Cukurova et al., 2019), retroalimentación de conocimientos (Popenici \& Kerr, 2017) y tutorías inteligentes (Sharma, Giannakos \& Dillenbourg, 2020). 


\section{Revistas científicas en el área de la IA de acceso libre}

La búsqueda realizada en el portal de SCImago Journal \& Country Rank, refleja el incremento de revistas científicas en el área de la IA desde el año 2010 hasta el año 2019 en un 200\%. La figura 1 detalla la aparición de revistas científicas por año.

Figura 1

Número de revistas científicas por año

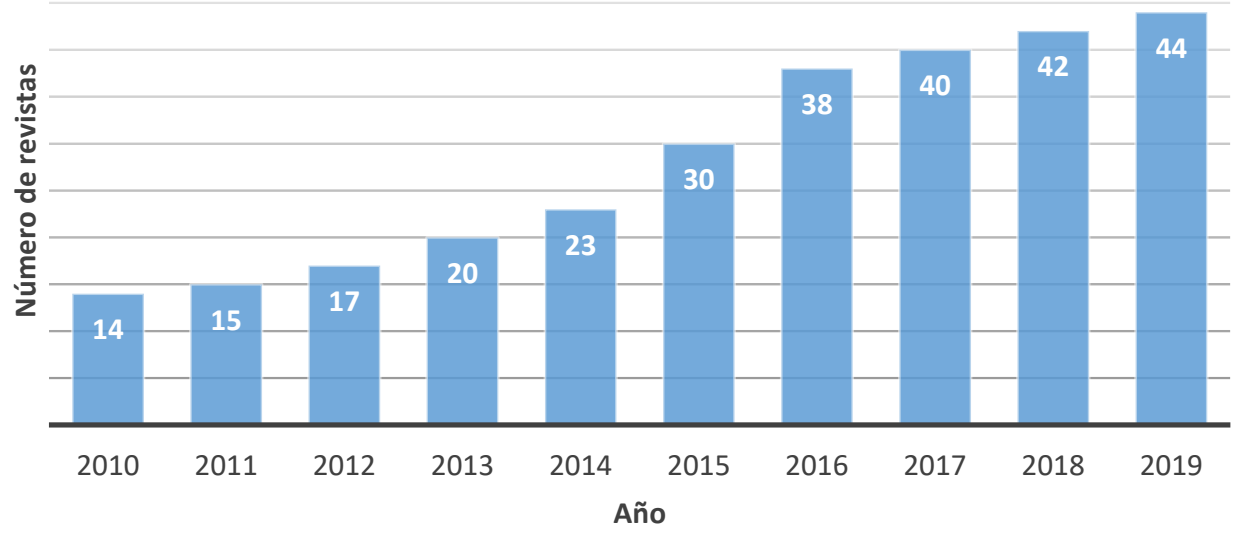

Fuente: elaboración propia

En el año 2010 se contaba con 14 revistas científicas, para el año 2015 excedió el doble llegando a 30 revistas, y en el año 2019 se triplicó este número inicial de revistas científicas, llegando a existir 44 revistas que publican investigaciones utilizando la IA como herramienta generadora de conocimientos.

De otro lado, en la búsqueda realizada en el portal de SCImago Journal \& Country Rank, se evidenció que las 44 revistas científicas existentes en el año 2019 se encuentran distribuidas en 18 países. En la tabla 2 se puede apreciar los nombres de las revistas, el cuartil en que se ubica y el país a la que pertenece. 


\section{Tabla 2}

Países con revistas científicas en el área de la IA

\begin{tabular}{|c|c|c|}
\hline País & $\begin{array}{l}\mathrm{N}^{\circ} \text { de } \\
\text { revistas }\end{array}$ & Nombre de la revista y cuartil en que se encuentra \\
\hline Estados Unidos & 7 & $\begin{array}{l}\text { Journal of Machine Learning Research (Q1), IEEE Robotics } \\
\text { and Automation Letters (Q1), Journal of Artificial } \\
\text { Intelligence Research (Q1), Computational Linguistics (Q2), } \\
\text { International Journal of Advanced Robotic Systems (Q3), } \\
\text { Statistics, Optimization and Information Computing (Q3), } \\
\text { Journal of Computer Science (Q4). }\end{array}$ \\
\hline Suiza & 5 & $\begin{array}{l}\text { Frontiers in Neurorobotics (Q2), Frontiers Robotics AI (Q2), } \\
\text { Frontiers in ICT (Q3), Robotics (Q3), ROBOMECH Journal } \\
\text { (Q3). }\end{array}$ \\
\hline Reino Unido & 4 & $\begin{array}{l}\text { i-Perception (Q2), Systems Science and Control Engineering } \\
\text { (Q3), Expert Systems with Applications: X, IET Cyber- } \\
\text { Physical Systems: Theory and Applications. }\end{array}$ \\
\hline China & 3 & $\begin{array}{l}\text { Computational Visual Media (Q2), International Journal of } \\
\text { Intelligent Systems and Applications (Q3), Fuzzy } \\
\text { Information and Engineering (Q3). }\end{array}$ \\
\hline Corea del Sur & 3 & $\begin{array}{l}\text { ICT Express (Q2), International Journal of Fuzzy Logic and } \\
\text { Intelligent Systems (Q3), Journal of Cognitive Science (Q4). }\end{array}$ \\
\hline Alemania & 3 & $\begin{array}{l}\text { Journal of Artificial Intelligence and Soft Computing } \\
\text { Research (Q2), Paladyn (Q3), Journal of Intelligent Systems } \\
\text { (Q4). }\end{array}$ \\
\hline Polonia & 3 & $\begin{array}{l}\text { Bulletin of the Polish Academy of Sciences: Technical } \\
\text { Sciences, Journal of Automation (Q3), Mobile Robotics and } \\
\text { Intelligent Systems (Q4), Computer Science (Q4). }\end{array}$ \\
\hline Federación Rusa & 2 & Cybernetics and Physics (Q3), SPIIRAS Proceedings (Q4). \\
\hline Irán & 2 & $\begin{array}{l}\text { Iranian Journal of Fuzzy Systems (Q2), Journal of } \\
\text { Information Systems and Telecommunication (Q4). }\end{array}$ \\
\hline República Checa & 2 & Kybernetika (Q3), Mendel (Q4). \\
\hline España & 2 & Inteligencia Artificial (Q4), Lingüística (Q4). \\
\hline Indonesia & 2 & $\begin{array}{l}\text { International Journal of Advances in Intelligent Informatics } \\
\text { (Q4), IAES International Journal of Artificial Intelligence } \\
\text { (Q4). }\end{array}$ \\
\hline
\end{tabular}

Países Bajos $\quad 1 \quad$ Procedia Manufacturing (Q2).

Egipto 1 Applied Computational Intelligence and Soft Computing (Q3). 


\begin{tabular}{lll}
$\begin{array}{l}\text { Eslovenia } \\
\text { India }\end{array}$ & 1 & $\begin{array}{l}\text { Informatica (Q4). } \\
\text { International Journal of Computer Information Systems and } \\
\text { Industrial Management Applications (Q4). }\end{array}$ \\
Taiwán & 1 & $\begin{array}{l}\text { International Journal of Automation and Smart Technology } \\
(\mathrm{Q} 4) .\end{array}$ \\
Bahréin & 1 & $\begin{array}{l}\text { International Journal of Computing and Digital Systems } \\
(\mathrm{Q} 4) .\end{array}$ \\
\hline
\end{tabular}

Fuente: elaboración propia

El país que lidera la tabla 2 con siete revistas científicas en el área de la IA es Estados Unidos, le sigue Suiza con cinco, el Reino Unido con cuatro, China y Corea del Sur con tres revistas cada uno. Estos cinco países poseen el 50\% de las revistas científicas en el área de la IA que existen en el mundo en el año 2019.

Asimismo, de las 44 revistas científicas en el área de la IA que se encontró en el portal de SCImago Journal \& Country Rank, no todas se encuentran en el ranking de revistas por cuartiles. En el año 2010, de las 14 revistas científicas existentes, dos de ellas no se encontraban en este ranking, lo cual equivale a un 14.29\%. En el año 2019, de las 44 revistas científicas existentes, solo dos de ellas no se encontraban en este ranking, lo cual equivale a un $4.55 \%$. La tabla 3 detalla las revistas científicas y el año en el que no se encontraban en el ranking por cuartiles 


\section{Tabla 3}

Revistas científicas en el área de la IA que no están en el ranking por cuartiles

\begin{tabular}{ll}
\hline Año & Revistas que no están en el ranking SJR \\
\hline 2010 & Mendel, i-Perception. \\
2011 & Mendel, International Journal of Automation and Smart Technology. \\
2012 & Mendel, Lingüística, Robotics. \\
2013 & Mendel, Journal of Information Systems and Telecommunication, Robotics, \\
& Statistics, Optimization and Information Computing, Systems Science and \\
& Control Engineering.
\end{tabular}

2014 Mendel, Frontiers in ICT, Fuzzy Information and Engineering, ROBOMECH Journal, Robotics.

2015 Mendel, Computational Visual Media, Frontiers in ICT, Frontiers Robotics AI, ICT Express, International Journal of Advances in Intelligent Informatics, International Journal of Computer Information Systems and Industrial Management Applications, Journal of Automation, Mobile Robotics and Intelligent Systems, Procedia Manufacturing, Robotics.

2016 Mendel, Applied Computational Intelligence and Soft Computing, Computational Visual Media, Cybernetics and Physics, Frontiers in ICT, Frontiers Robotics AI, IEEE Robotics and Automation Letters, International Journal of Advances in Intelligent Informatics, International Journal of Intelligent Systems and Applications, Journal of Artificial Intelligence and Soft Computing Research, Journal of Cognitive Science, Paladyn, Robotics, SPIIRAS Proceedings.

2017 Mendel, Applied Computational Intelligence and Soft Computing, Computational Visual Media, Computer Science, Frontiers in ICT, Frontiers Robotics AI, IEEE Robotics and Automation Letters, International Journal of Advances in Intelligent Informatics, International Journal of Fuzzy Logic and Intelligent Systems, Robotics.

2018 Mendel, Frontiers in ICT, IAES International Journal of Artificial Intelligence, IEEE Robotics and Automation Letters, International Journal of Advances in Intelligent Informatics, International Journal of Computing and Digital Systems.

2019 Expert Systems with Applications: X, IET Cyber-Physical Systems: Theory and Applications

Fuente: elaboración propia 
En la tabla 4 se describen los datos de las dos revistas que no están dentro del ranking por cuartiles en el año 2019.

Tabla 4

Revistas que no están en el ranking en el año 2019

\begin{tabular}{|c|c|c|c|c|}
\hline $\begin{array}{l}\text { Nombre de la } \\
\text { revista }\end{array}$ & Creación & Área temática y categoría & Editor & ISSN \\
\hline $\begin{array}{l}\text { Expert } \\
\text { Systems with } \\
\text { Applications: } \\
\text { X }\end{array}$ & 2019 & $\begin{array}{l}\text { Ciencias de la computación: } \\
\text { inteligenciarartificial, } \\
\text { aplicaciones informáticas. } \\
\text { Ingeniería: todas las áreas. }\end{array}$ & Elsevier Ltd. & 25901885 \\
\hline $\begin{array}{l}\text { IET Cyber- } \\
\text { Physical } \\
\text { Systems: } \\
\text { Theory and } \\
\text { Applications }\end{array}$ & 2019 & $\begin{array}{l}\text { Ciencias de la computación: } \\
\text { inteligencia artificial, redes } \\
\text { informáticas } \\
\text { comunicaciones, aplicaciones } \\
\text { informáticas, sistemas de } \\
\text { información. } \\
\text { Ingeniería: ingeniería eléctrica } \\
\text { y electrónica. }\end{array}$ & $\begin{array}{l}\text { Institución de } \\
\text { Ingeniería y } \\
\text { Tecnología. }\end{array}$ & 23983396 \\
\hline
\end{tabular}

Fuente: elaboración propia

El portal de SCImago Journal \& Country Rank, también nos permite conocer la calidad de las publicaciones científicas, la figura 2 nos muestra el número de revistas agrupadas en cuartiles Q1, Q2, Q3 y Q4 durante el periodo 2010-2019.

\section{Figura 2}

Número de revistas por cuartiles

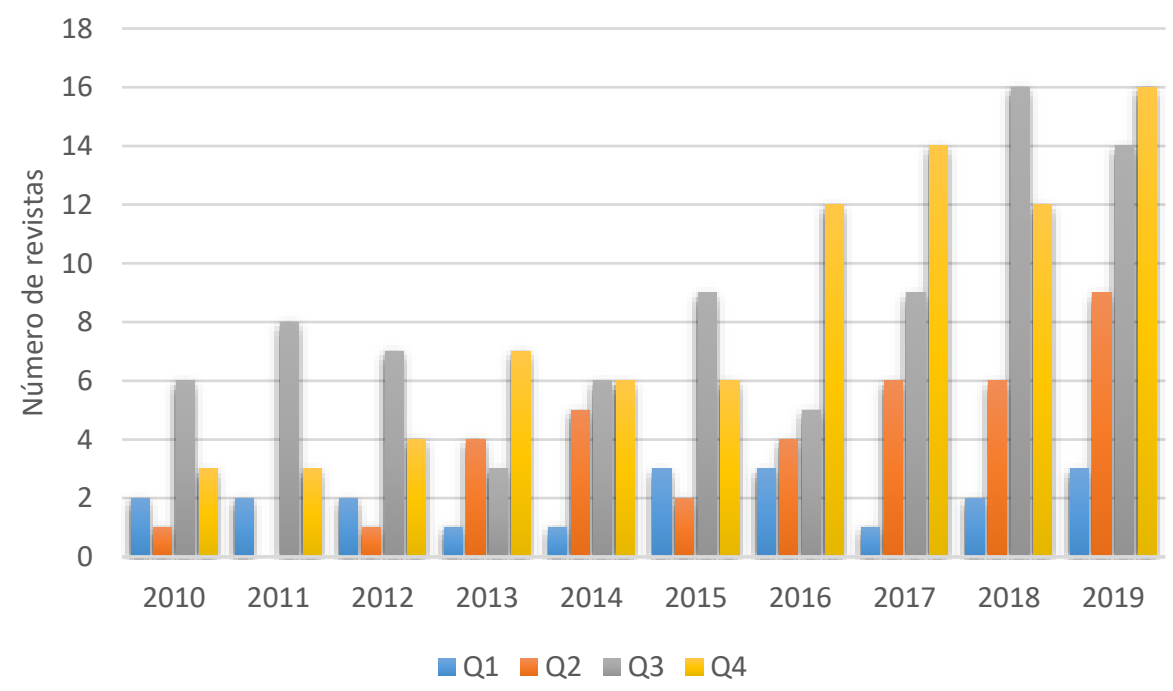

Fuente: elaboración propia 
Se puede visualizar que el cuartil Q1 es el que almacena menos revistas en el año 2019, solamente tres revistas se encuentran en el primer cuartil: Journal of Machine Learning Research, IEEE Robotics and Automation Letters y Journal of Artificial Intelligence Research. En la tabla 5 describimos los datos de estas revistas.

\section{Tabla 5}

Revistas ubicadas en el primer cuartil en el 2019

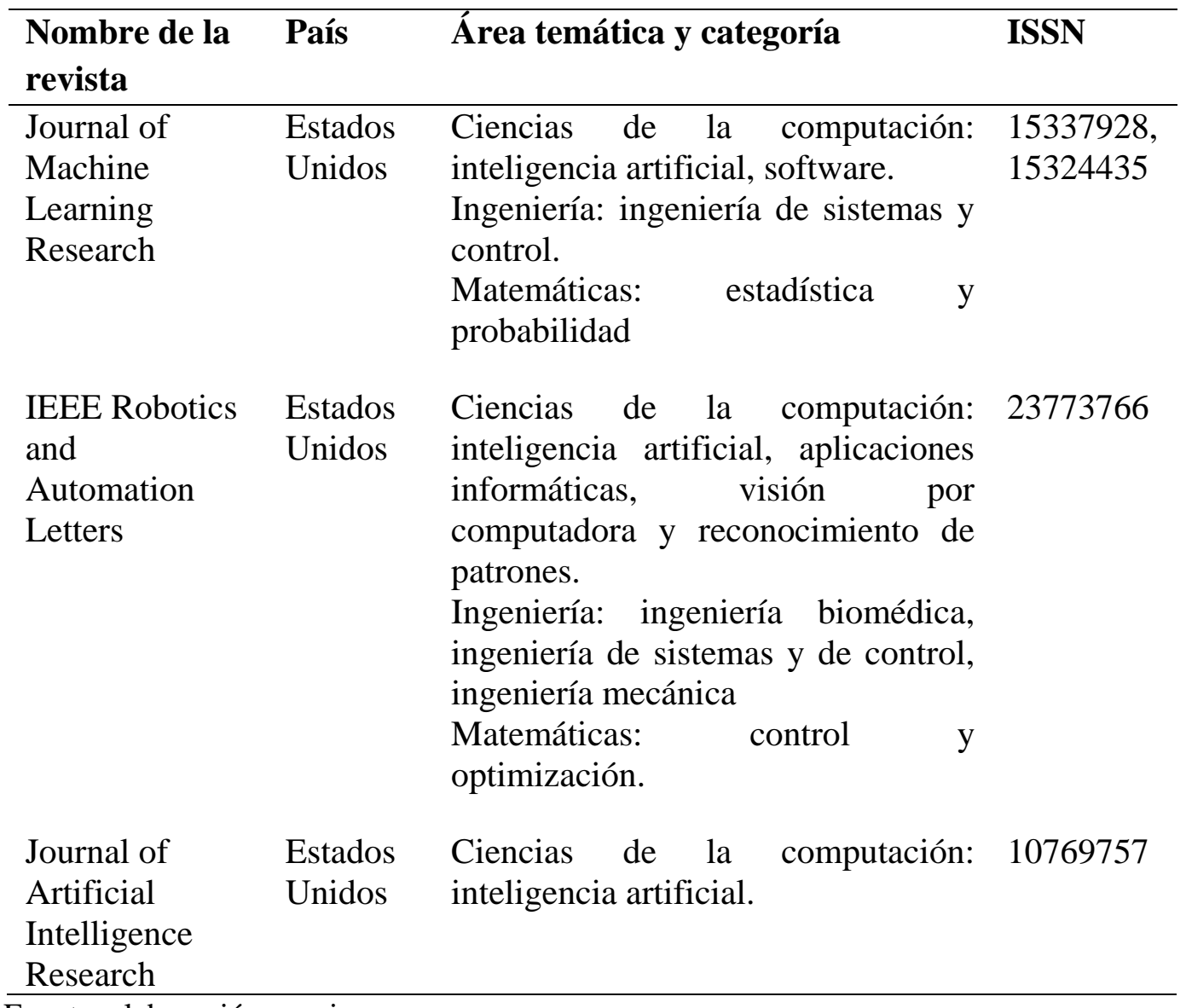

Fuente: elaboración propia

Los cuartiles que más revistas científicas almacenan son el Q3 y el Q4. De las 44 revistas existentes en el año 2019, el 6.82\% se encuentran en el cuartil Q1, el 20.45\% se encuentran en el cuartil Q2, el 31.82\% en el cuartil Q3 y el 36.36\% en el cuartil Q4. La figura 3 nos ilustra el número de documentos por año que publicaron las revistas científicas en el área de la IA. 


\section{Figura 3}

Número de documentos publicados por año

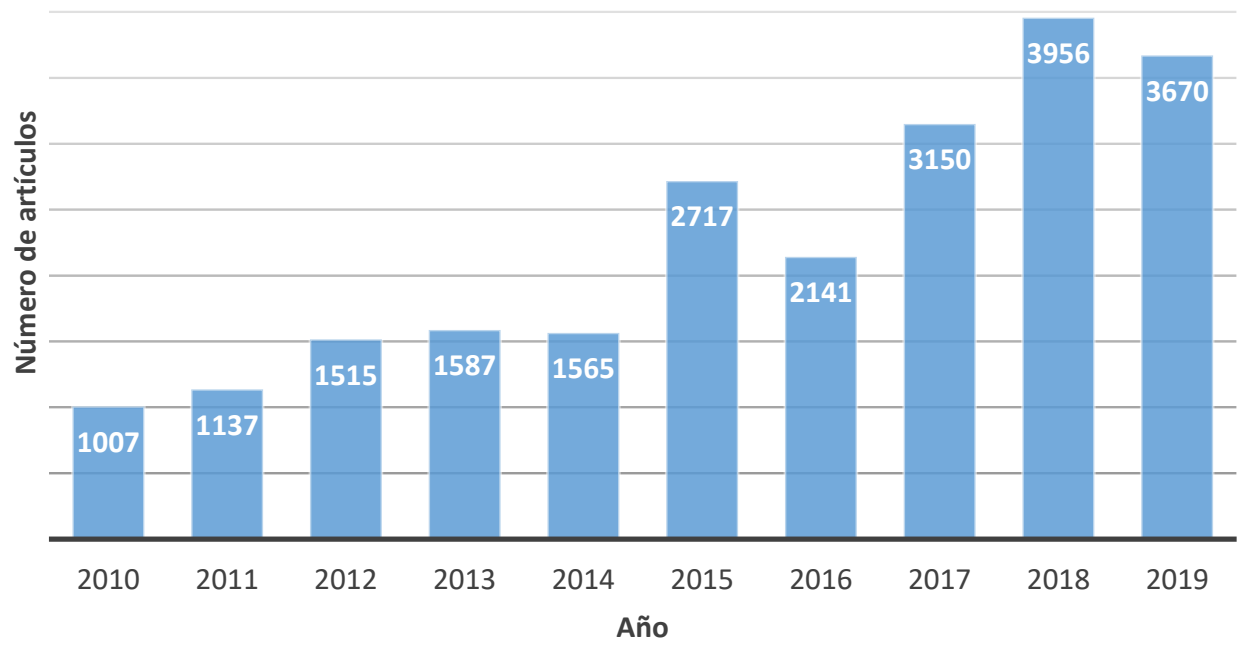

Fuente: elaboración propia

Durante el periodo 2010-2019 se publicaron 22445 documentos científicos en el área de la IA. El año 2018 registra el máximo número de documentos publicados, siendo este 3956. Le sigue el año 2019 con 3670 publicaciones, y en tercer lugar el año 2017 con 3150 publicaciones.

\section{Discusión}

En esta época de pandemia, la educación a distancia, remota o no presencial, se impuso como una alternativa de solución a la necesidad de continuar con el proceso educativo, la cual es posible gracias a las herramientas que ofrece la tecnología. Por ello, es necesario ahondar en esta línea de investigación puesto que es el futuro de la educación moderna, ya sea en un entorno presencial, semipresencial o virtual, garantizando de esta manera una educación de calidad en países desarrollados, subdesarrollados y en vía de desarrollo (Cukurova et al., 2019; Matas-Terrón et al., 2020).

El año 2019, los 3,670 documentos publicados sobre IA y educación en las 44 revistas científicas descritas en la Tabla 2, dan cuenta del auge que ha tomado esta disciplina, y exponen la necesidad de replicar esta conducta en investigadores de América del Sur. La educación a distancia o no presencial que se viene desarrollando a causa de esta pandemia, es posible gracias a los aportes de la IA a lo largo de las décadas (Moreira et al., 2019; Zawacki-Richter et al., 2019). 
En la revisión bibliográfica realizada, no se encontraron revistas científicas en el área de la IA en América del Sur, lo cual no implica la inexistencia de artículos científicos en esta materia publicados en revistas relacionadas a otras áreas que no se encuentran en el portal SCImago Journal \& Country Rank en esta parte del continente. Surge la necesidad de fortalecer y promover el empleo equitativo e inclusivo de la IA en el sector educativo, con la finalidad de alcanzar el logro de competencias necesarias para la vida y el campo laboral en América del Sur (Wang \& Zhan, 2021), promover la investigación bajos los principios de la ética y la transparencia que priman en la educación (Soong \& Ho, 2021).

Promover la utilización de la IA en la educación, implica poner a esta al servicio de la investigación, gestión, administración e innovación educativa. Un trabajo arduo que conlleva el empoderamiento de docentes y estudiantes, consecuentemente una nueva forma de enseñanza y evaluación de resultados, promoviendo la equidad de aprendizajes a lo largo de la vida (Rampton, Mittelman \& Goldhahn, 2020; Renz \& Hilbig, 2020).

No obstante, una limitación presente en la búsqueda de información en el portal SCImago Journal \& Country Rank, fue el acceso abierto a los artículos. Identificar los aportes de la IA, y analizar el papel que desempeñará en la educación post Covid-19, en cierto momento resultó difícil, sin embargo esto no fue obstáculo para conocer los índices de impacto de las publicaciones en el área de la IA en los últimos 10 años. Debido a que SCImago Journal Rank evalúa la métrica de impacto de las publicaciones basado en la información incluida en la base de datos de Scopus, en esta investigación no se ha tenido en consideración publicaciones de revistas científicas indexadas únicamente en: Web of Science, Wiley, IEEE Xplore, Mathematical Reviews, Medline Taylor \& Francis Group, EconLit, ProQuest, Bentham Science, SciELO, DOAJ, Redalyc, Latindex, entre otras. No hay duda de la existencia de revistas científicas indexadas en las bases de datos mencionadas anteriormente, que están relacionadas a la IA y educación, cuyas investigaciones aportan conocimientos valiosos al sector educación, pero esto ya es motivo de otra investigación, particularmente teniendo en consideración la región de América del Sur.

\section{Conclusiones}

Esta investigación ha recopilado producciones científicas de revistas pertenecientes al área de la IA encontradas en el portal SCImago Journal \& Country Rank. A partir del año 2010 se refleja un aumento progresivo de revistas científicas en el área de la IA. En el portal de SCImago Journal 
\& Country Rank se pueden visualizar 18 países que cuentan con revistas científicas en el área de la IA en la actualidad, siendo Estados Unidos el país con mejor ranking de revistas en el área de la IA. En una educación post-Covid-19, la IA jugará un papel muy importante, donde sistemas de gestión de aprendizajes, tutores inteligentes, plataformas interactivas, entre otras herramientas, garantizaran la calidad educativa en el proceso de enseñanza y aprendizaje, se avizora una educación que traspasará fronteras económicas, políticas, sociales, culturales, de tiempo y lugar. Finalmente, se recomienda innovar en educación investigaciones que involucren como línea de investigación de la inteligencia artificial a los sistemas inteligentes, contenidos digitales interactivos, realidad virtual y realidad aumentada, redes neuronales, entre otras; a fin de dar solución a diversos problemas que este sector presenta.

\section{Referencias}

Amershi, S., Arksey, N., Carenini, G., Conati, C., Mackworth, A., Maclaren, H., y Poole, D. (2005). Designing CIspace: Pedagogy and usability in a learning environment for AI. Proceedings of the 10th Annual SIGCSE Conference on Innovation and Technology in Computer Science Education - ITiCSE '05, 178. Capacrica, Portugal: ACM Press. https://doi.org/10.1145/1067445.1067495

Aranda-Corral, G. A., Calmet, J., y Martín-Mateos, F. J. (Eds.). (2014). Artificial Intelligence and Symbolic Computation. Cham: Springer International Publishing. https://doi.org/10.1007/978-3-319-13770-4

Arevalillo-Herráez, M., Moreno-Picot, S., y Cavero-Millán, V. (2011). A computer architecture to use AIBO robots to teach Artificial Intelligence. Revista Iberoamericana de Tecnologias del Aprendizaje, $\quad 6(1), \quad 1-9$. http://rita.det.uvigo.es/index.php?content=Num_Pub\&idiom=Es\&visualiza=1\&volumen= $6 \&$ numero $=1 \&$ orden $=$ desc

Benítez-Restrepo, M. (2020). Cultura académica y enseñanza-aprendizaje en educación superior. Revisión de literatura. Magis, Revista Internacional de Investigación en Educación, 13, 123. https://doi.org/10.11144/Javeriana.m13.caea

Benkovič, J., Bohanec, M., Rajkovič, V., y Vrtačnik, M. (1997). Knowledge-Based Evaluation of Higher Education Institutions. 6th IFAC Symposium on Automated Systems Based on Human Skill 1997 (Joint Design of Technology and Organisation), Kranjska gora, 
Slovenia, 17-19 September, 30(24), 157-160. https://doi.org/10.1016/S14746670(17)42247-6

Boden, M. A. (1984). Impacts of artificial intelligence. Futures, 16(1), 60-70. https://doi.org/10.1016/0016-3287(84)90007-7

Booth, A., Sutton, A., y Papaioannou, D. (2021). Systematic Approaches to a Successful Literature Review (2nd ed.). Reino Unido: The University of Sheffield. https://us.sagepub.com/enus/nam/systematic-approaches-to-a-successful-literature-review/book244586

Brazdil, P., y Jorge, A. (Eds.). (2001). Progress in Artificial Intelligence: Knowledge Extraction, Multi-agent Systems,Logic Programming, and Constraint Solving. Berlin, Heidelberg: Springer Berlin Heidelberg. https://doi.org/10.1007/3-540-45329-6

Brown, J. S., y Burton, R. R. (1978). A paradigmatic example of an artificially intelligent instructional system. International Journal of Man-Machine Studies, 10 (3), 323-339. https://doi.org/10.1016/S0020-7373(78)80050-9

Brusilovsky, P., y Peylo, C. (2003). Adaptive and intelligent web-based educational systems. International Journal of Artificial Intelligence in Education, 13 (2-4), 159-172. https://iaied.org/journal/960

Caspar, P. (1990). Investing in intelligence? Futures, 22 (7), 710-729. https://doi.org/10.1016/0016-3287(90)90028-G

Castrillón, O. D., Sarache, W., y Ruiz-Herrera, S. (2020). Prediction of academic performance using artificial intelligence techniques. Formacion Universitaria, 13 (1), 93-102. https://doi.org/10.4067/S0718-50062020000100093

Cerda, G., Pérez, C., Elipe, P., Casas, J. A., y Del Rey, R. (2019). Convivencia escolar y su relación con el rendimiento académico en alumnado de Educación Primaria. Revista de Psicodidáctica, 24 (1), 46-52. https://doi.org/10.1016/j.psicod.2018.05.001

Chacón-Vargas, É., y Roldán-Villalobos, G. (2021). Factors affecting the academic performance of freshmen in the ITCR general mathematics course. Uniciencia, 35 (1). https://doi.org/10.15359/RU.35-1.16

Chandrasekaran, B. (1975). Artificial Intelligence: The Past Decade. En M. Rubinoff y M. C. Yovits (Eds.), Advances in Computers (Vol. 13, pp. 169-232). https://doi.org/10.1016/S0065-2458(08)60658-0 
Coldwell, D. (1995). Artificial intelligence and mute autistic children. Journal of Microcomputer Applications, 18 (4), 305-311. https://doi.org/10.1016/S0745-7138(05)80029-6

Cukurova, M., Kent, C., y Luckin, R. (2019). Artificial intelligence and multimodal data in the service of human decision-making: A case study in debate tutoring. British Journal of Educational Technology, 50 (6), 3032-3046. https://doi.org/10.1111/bjet.12829

Cukurova, M., Luckin, R., y Clark-Wilson, A. (2019). Creating the golden triangle of evidenceinformed education technology with EDUCATE. British Journal of Educational Technology, 50 (2), 490-504. https://doi.org/10.1111/bjet.12727

Cukurova, M., Luckin, R., y Kent, C. (2020). Impact of an Artificial Intelligence Research Frame on the Perceived Credibility of Educational Research Evidence. International Journal of Artificial Intelligence in Education, 30 (2), 205-235. https://doi.org/10.1007/s40593-01900188-W

Gable, A., y Page, C. V. (1980). The use of Artificial Intelligence techniques in Computer-Assisted Instruction: An overview. International Journal of Man-Machine Studies, 12 (3), 259-282. https://doi.org/10.1016/S0020-7373(80)80028-9

Garrido, A. (2010). Mathematics and Artificial Intelligence, two branches of the same tree. Innovation and Creativity in Education, 2 (2), 1133-1136. https://doi.org/10.1016/j.sbspro.2010.03.160

Goldstein, I., y Papert, S. (1977). Artificial intelligence, language, and the study of knowledge. Cognitive Science, 1 (1), 84-123. https://doi.org/10.1016/S0364-0213(77)80006-2

González-García, N., Sánchez-García, A. B., Nieto-Librero, A. B., y Galindo-Villardón, M. P. (2019). Actitud y enfoques de aprendizaje en el estudio de la Didáctica General. Una visión multivariante. Revista de Psicodidáctica, $24 \quad$ (2), 154-162. https://doi.org/10.1016/j.psicod.2019.02.002

Guzmán-Zamora, N., y Gutiérrez-García, R. A. (2020). School motivation: Academic goals, attributional styles and academic performance in middle education students. Archivos Venezolanos de Farmacologia y Terapeutica, $39 \quad$ (3), 290-295. http://www.revistaavft.com/images/revistas/2020/avft_3_2020/9_motivacion.pdf

Incio, F. A., Capuñay, D. L., Estela, R. O., Delgado, J. A., y Vergara, S. E. (2021). Diseño e implementación de una red neuronal artificial para predecir el rendimiento académico en 
estudiantes de Ingeniería Civil de la UNIFSLB. Revista Veritas et Scientia, 10 (1), $107-$ 117. https://doi.org/10.47796/ves.v10i1.464

Jones, M. (1985). Applications of artificial intelligence within education. Computers \& Mathematics with Applications, 11 (5), 517-526. https://doi.org/10.1016/08981221(85)90054-9

Kelly, A. E., Sleeman, D. H., y Gilhooly, K. J. (1993). Artificial intelligence in education: Using state space search and heuristics in mathematics instruction. International Journal of ManMachine Studies, 38 (4), 725-746. https://doi.org/10.1006/imms.1993.1034

Lillehaug, S.-I., y Lajoie, S. P. (1998). AI in medical education: Another grand challenge for medical informatics. Artificial Intelligence in Medicine, 12 (3), 197-225. https://doi.org/10.1016/S0933-3657(97)00054-7

Luckin, R., y Cukurova, M. (2019). Designing educational technologies in the age of AI: A learning sciences-driven approach. British Journal of Educational Technology, 50 (6), 2824-2838. Scopus. https://doi.org/10.1111/bjet.12861

Matas-Terrón, A., Leiva-Olivencia, J. J., Franco-Caballero, P. D., y García-Aguilera, F. J. (2020). Validity of the «Big Data tendency in education»scale as a tool helping to reach inclusive $\begin{array}{llll}\text { social development. } & \text { Sustainability } & \text { (Switzerland), } & 12\end{array}$ https://doi.org/10.3390/su12135470

Moreira, F., Ferreira, M. J., Pereira, C. S., Gomes, A. S., Collazos, C., y Escudero, D. F. (2019). ECLECTIC as a learning ecosystem for higher education disruption. Universal Access in the Information Society, 18 (3), 615-631. https://doi.org/10.1007/s10209-019-00682-x

Naughton, J. (1987). 7-Artificial intelligence and education and training. En Artificial Intelligence (pp. 77-82). Pergamon. https://doi.org/10.1016/B978-0-08-034112-5.50012-7

Pham, D. T., y Pham, P. T. N. (1999). Artificial intelligence in engineering. International Journal of Machine Tools and Manufacture, 39 (6), 937-949. https://doi.org/10.1016/S08906955(98)00076-5

Pinto, P. M., Fernández, Y. O., y Cabezas, N. G. (2020). Intercultural education in the process of educational humanization of college students: Influences on academic achievement. Interciencia, $45 \quad$ (4), 201-208. https://www.interciencia.net/wpcontent/uploads/2020/04/04_6645_E_Martinez_v45n4_8.pdf 
Popenici, S. A. D., y Kerr, S. (2017). Exploring the impact of artificial intelligence on teaching and learning in higher education. Research and Practice in Technology Enhanced Learning, 12 (1). https://doi.org/10.1186/s41039-017-0062-8

Rampton, V., Mittelman, M., y Goldhahn, J. (2020). Implications of artificial intelligence for medical education. The Lancet Digital Health, 2 (3), e111-e112. https://doi.org/10.1016/S2589-7500(20)30023-6

Randolph, J. (2019). A Guide to Writing the Dissertation Literature Review. Practical Assessment, Research, and Evaluation, 14 (1). https://doi.org/10.7275/b0az-8t74

Renz, A., y Hilbig, R. (2020). Prerequisites for artificial intelligence in further education: Identification of drivers, barriers, and business models of educational technology companies. International Journal of Educational Technology in Higher Education, 17 (1). https://doi.org/10.1186/s41239-020-00193-3

Rowe, N. C. (1980). Inductive common ground tutoring. Computers \& Education, 4 (3), 177-187. https://doi.org/10.1016/0360-1315(80)90030-5

Salchenberger, L. (1989). A strategy for integrating artificial intelligence technology into a graduate business curriculum. Education and Computing, 5 (3), 189-196. https://doi.org/10.1016/S0167-9287(89)80042-1

Schaufelberger, W. (1993). Low cost control education software for MS-DOS PCs. Automatica, 29 (6), 1501-1516. https://doi.org/10.1016/0005-1098(93)90013-J

Self, J. A. (1979). Student models and artificial intelligence. Computers \& Education, 3 (4), 309312. https://doi.org/10.1016/0360-1315(79)90008-3

Sharma, K., Giannakos, M., y Dillenbourg, P. (2020). Eye-tracking and artificial intelligence to enhance motivation and learning. Smart Learning Environments, 7 (1). https://doi.org/10.1186/s40561-020-00122-x

Siemer, J., y Angelides, M. C. (1998). A comprehensive method for the evaluation of complete intelligent tutoring systems. Decision Support Systems, 22 (1), 85-102. https://doi.org/10.1016/S0167-9236(97)00033-X

Smith, F. J., Sullivan, M., Collis, J., y Loughlin, S. (1997). Applying Artificial Intelligence in Physical Chemistry. En J. R. Sabin, M. C. Zerner, J. Karwowski, y M. Karelson (Eds.), Advances in Quantum Chemistry (Vol. 28, pp. 319-328). Academic Press. https://doi.org/10.1016/S0065-3276(08)60224-5 
Soong, T. K., y Ho, C.-M. (2021). Artificial intelligence in medical OSCEs: Reflections and future developments. Advances in Medical Education and Practice, 12, 167-173. https://doi.org/10.2147/AMEP.S287926

Vila, E. M. S., y Penín, M. L. (2007). Introduction to special issue AI techniches applied in education. Inteligencia Artificial, 11(33), 7-12. http://journal.iberamia.org/public/iaold/articles/522/article\%20\%281\%29.pdf

Wang, J., y Zhan, Q. (2021). Visualization Analysis of Artificial Intelligence Technology in Higher Education Based on SSCI and SCI Journals from 2009 to 2019. International Journal of Emerging Technologies in Learning, 16 20-33. https://doi.org/10.3991/ijet.v16i08.18447

Wasson, B. (1997). Advanced educational technologies: The learning environment. Norwegian Perspectives on Computing in Complex Domains, 13 (4), 571-594. https://doi.org/10.1016/S0747-5632(97)00027-7

Zawacki-Richter, O., Marín, V. I., Bond, M., y Gouverneur, F. (2019). Systematic review of research on artificial intelligence applications in higher education - where are the educators? International Journal of Educational Technology in Higher Education, 16 (1). https://doi.org/10.1186/s41239-019-0171-0

Zhang, J. (2005). Intelligent Tutoring Systems: Research Status and Its Development in China. 2005 International Conference on Natural Language Processing and Knowledge Engineering, 683-689. Wuhan, China: IEEE. https://doi.org/10.1109/NLPKE.2005.1598823 